\title{
Comment to: "Diagnostic Benefit of the Detection of Mitotic Figures in Endometriotic Lesions"
}

\author{
Kommentar zu „Diagnostischer Vorteil des Nachweises \\ von Mitosefiguren in endometriotischen Läsionen“
}

(C) 9 (9)

\author{
Authors \\ Karl Werner Schweppe ${ }^{1,2}$, Klaus Bühler ${ }^{1,3}$ \\ Affiliations \\ 1 Stiftung Endometriose-Forschung, Westerstede, Germany \\ 2 Obst. \& Gynec., Endometriose-Zentrum Ammerland, \\ Westerstede, Germany \\ 3 klin.-wissenschaftl. Endometriosezentrum der Univ.- \\ Kliniken des Saarlandes Endometriose-Sprechstunde - \\ Frauenärzte am Staden, Saarbrücken, Germany
}

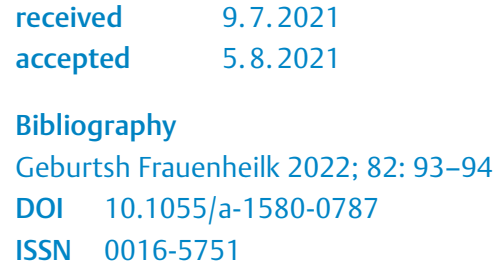

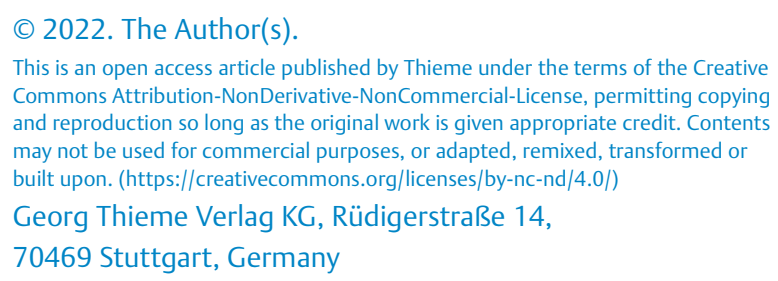

The publication: "Diagnostic Benefit of the Detection of Mitotic Figures in Endometriotic Lesions" by M. Wetzk et al. is of great significance and importance insofar as it shows how the histological diagnosis of active, endocrinologically modulated endometriosis can be described more precisely with a simple method. The mitosis index as an unpretentious morphologic parameter of the activity and proliferation potential of endometriotic implants is of great implication for subsequent treatment and probably for the prognosis of the disease i.e. reaction to medical treatment or risk of recurrences. In the endometriosis centres of excellence certified by the Scientific Endometriosis Research Foundation (SEF) and the European Endometriosis League (EEL), where patients more and more turn to for further therapy or for a second opinion after the detection of endometriosis, we increasingly see only the terse statement of endometriosis in the patho-histological report. However, it does not cost the pathologist much more time to also describe characteristic features of endometriotic implants in the histological report.

In a clinical study [1] with 544 endometriosis patients, macroscopic and microscopic criteria were used to classify endometriosis into active and inactive. Criteria for the activity of the glandular epithelium were high, endometrial-like differentiation, mitoses, eutrophy and signs of hormonal influence, similar to the changes in the eutopic endometrium during the menstrual cycle. Signs of inactivity were cubic, flattened epithelium, lack of mitoses and lack of endocrine modulation, as well as atrophy. In assessing the stroma, good vascularization, edema, bleeding and signs of inflammation were criteria for activity and fibrosis, siderophages and lack of vascularization signs for inactivity. It was found that in the group of pain patients with active endometriosis, the recurrence rate was significantly lower and the recurrence-free interval significantly longer after three-phase therapy compared to laparoscopic surgery alone. In the case of inactive manifestations of endometriosis, no differences could be observed, so that this group of patients had no advantage from the six-month medical treatment and re-laparoscopy.

But also the surgeon is required to describe the endometriosis lesions in more detail. Even the macroscopic aspects, color and type of the lesion are significant in terms of the endometriotic activity. In the above-mentioned study, vesicles-shaped or polypoid peritoneal implants that were colored red with perifocal bleeding and vascularization were judged to be active; brown, black or flattened foci and cicatricial thickenings of the peritoneum, on the other hand, as inactive form of endometriosis.

The importance of these criteria, which are additive to the rASRM classification, has also been pointed out by other investiga- 
tors [2]. Furthermore, a sufficient morphological assessment of endometriosis biopsies also requires the consideration of atypia and dysplasia. In a review of a large series of studies, approximately $8 \%$ of endometrioses contain endometriosis with cellular atypia [3]. Studies have shown that the risk of developing endometroid or clear cell ovarian cancer is increased by a factor of 23 in women with endometriosis and therefore it is necessary to search specifically for precursors [4]. Morphological examinations showed that there exists a continuous process of successive stages from normal epithelium in an endometrioma to cell atypia and subsequently to a possible invasive carcinoma [5].

As many of our patients suffer from endometriosis-related infertility we have to remember that - as we know from data of reproductive medicine -, after IVF therapy has been performed, the risk of malignancy is higher in endometriosis and in nulliparous patients $[6,7]$.

This means two things for the clinician:

1. cooperation with the pathologist and motivation that he comments on additive criteria such as activity, inflammation and atypia in the endometriosis biopsies and

2. the resulting individual treatment plan, which not only takes into account the severity and extent as well as localization of the disease, but also, depending on the morphological findings, a medical follow-up treatment, including a relapse prophylaxis, or merely a regular gynecological aftercare, similar to oncology.
Conflict of Interest

The authors declare that they have no conflict of interest.

\section{References}

[1] Schweppe KW. Aktive und inaktive Endometriose-eine prognose- und therapierelevante Differentialdiagnose. Zentralbl Gynakol 1999; 121: 330-335

[2] Bouquet de Joliniere J, Major A, Ayoubi JM et al. It Is Necessary to Purpose an Add-on to the American Classification of Endometriosis? This Disease Can Be Compared to a Malignant Proliferation While Remaining Benign in Most Cases. EndoGram ${ }^{\circledR}$ Is a New Profile Witness of Its Evolutionary Potential. Front Surg 2019; 6: 27

[3] Van Gorp T, Amant F, Neven P et al. Endometriosis and the development of malignant tumours of the pelvis: a review of literature. Best Pract Res Clin Obstet Gynaecol 2004; 18: 349-371

[4] Wei J], William J, Bulun S. Endometriosis and Ovarian Cancer: A Review of Clinical, Pathologic, and Molecular Aspects. Int J Gynecol Pathol 2011; 30: 553-568

[5] Mikhaleva LM, Davydov Al, Patsap Ol et al. Malignant Transformation and Associated Biomarkers of Ovarian Endometriosis: A Narrative Review. Adv Ther 2020; 37: 2580-2603

[6] Van Leeuwen FE, Klip H, Mooij TM et al. Risk of borderline and invasive ovarian tumours after ovarian stimulation for in vitro fertilization in a large Dutch cohort. Hum Reprod 2011; 26: 3456-3465

[7] Vassard D, Schmidt L, Glazer CH et al. Assisted reproductive technology treatment and risk of ovarian cancer-a nationwide population-based cohort study. Hum Reprod 2019; 34: 2290-2296 